\title{
Mechanical regulation of organ asymmetry in
} leaves

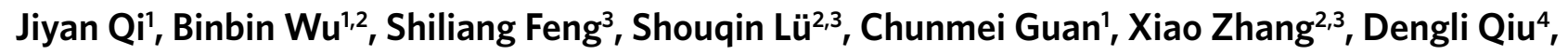 \\ Yingchun $\mathrm{Hu}^{5}$, Yihua Zhou ${ }^{1,2}$, Chuanyou Li ${ }^{1,2}$, Mian Long ${ }^{2,3 \star}$ and Yuling Jiao ${ }^{1,2 \star}$
}

\begin{abstract}
How appendages, such as plant leaves or animal limbs, develop asymmetric shapes remains a fundamental question in biology. Although ongoing research has revealed the genetic regulation of organ pattern formation, how gene activity ultimately directs organ shape remains unclear. Here, we show that leaf dorsoventral (adaxial-abaxial) polarity signals lead to mechanical heterogeneity of the cell wall, related to the methyl-esterification of cell-wall pectins in tomato and Arabidopsis. Numerical simulations predicate that mechanical heterogeneity is sufficient to produce the asymmetry seen in planar leaves. Experimental tests that alter pectin methyl-esterification, and therefore cell wall mechanical properties, support this model and lead to polar changes in gene expression, suggesting the existence of a feedback mechanism for mechanical signals in morphogenesis. Thus, mechanical heterogeneity within tissue may underlie organ shape asymmetry.
\end{abstract}

$\mathrm{D}$ uring development, gene expression patterns are established to direct the formation of biological shapes. Compared with gene expression patterns, our understanding of how genes regulate biological shapes remains rudimentary'. Organ growth involves physical processes. Emerging evidence indicates that mechanical stresses act as instructive signals to control morphogenesis in animals and in plants ${ }^{2,3}$. In plants, the mechanical constraints that arise from shared cell walls can orchestrate plant cell growth. Recent studies have shown that mechanical signals can regulate diverse aspects of development, such as cell division plane orientation $^{4}$, cell shape patterning ${ }^{5}$, organ initiation ${ }^{6}$, organ shape $e^{7}$ and plant architecture ${ }^{8}$. Although cells can rearrange their relative position in animals, tissue connectivity and associated stress may also help orchestrate growth in that kingdom?.

In plants, cell wall determines cell, and ultimately tissue, growth rate and direction. The cell wall allows plant cells to maintain high internal turgor pressure, preventing them from bursting. It is widely assumed that cell expansion results from irreversible yielding of the cell walls to high internal turgor pressure ${ }^{10,11}$. As an external matrix, the cell wall is composed of polysaccharides and proteins ${ }^{12,13}$. Growing cells are surrounded by the primary cell wall, which is mainly made up of pectin, cellulose and hemicellulose. Pectins are small polysaccharides synthesized by the cell mainly in a methylesterified form but subject to enzymatic de-methyl-esterification ${ }^{14}$. Pectin de-methyl-esterification reduces growth in the pollen tube, but increased de-methyl-esterification leads to increased cell wall extensibility and cell expansion in the shoot apex ${ }^{6,15}$. Cellulose reorientation may affect the mechanical anisotropy of cell walls and cellulose content affects wall elasticity ${ }^{16}$.

The leaf, as a representative plant lateral organ, provides an excellent model to study the establishment of organ asymmetry. Leaf primordia initiate as rounded bulges from the periphery of the shoot apical meristem (SAM), which maintains stem cells in the shoot apex. Soon after initiation, asymmetries become established. The establishment of adaxial-abaxial (dorsoventral) polarity has been proposed to condition establishment of the central-marginal axis and subsequent lateral expansion ${ }^{17}$. Extensive molecular genetic studies have identified a transcriptional regulatory network of genes that promote abaxial and adaxial fates and show adaxial or abaxial expression in the leaf ${ }^{18-23}$. Regulatory genes expressed in the abaxial domain suppress those expressed in the adaxial domain, and vice versa. Among others, REVOLUTA (REV) and related HD-ZIPIII genes, as well as the LOB-domain gene ASYMMETRIC LEAVES2 (AS2), are expressed in the adaxial domain to promote the adaxial cell fate ${ }^{24,25}$. On the other hand, FILAMENTOUS FLOWER (FIL) is expressed in the abaxial side ${ }^{26}$. It has long been proposed that the SAM produces a signal to promote leaf adaxial-abaxial patterning ${ }^{27}$. More recent work showed that transport of the phytohormone auxin from leaf primordia to the SAM leads to a transient low-auxin zone in the adaxial domain, which regulates leaf asymmetric development $^{28}$. How these adaxial- and abaxial-promoting genes regulate cell growth to change leaf primordium shape from round to flattened remains largely unknown.

In this study, we attempt to bridge the gap between gene expression patterns and biological shape. Our results show that leaf polarity signals lead to mechanical heterogeneity of the cell wall, which is related to the wall pectin methyl-esterification. Further numerical simulations, in combination with experimental validations, suggest that mechanical heterogeneity is sufficient to produce the planar leaf asymmetry. Thus, tissue-level mechanical heterogeneity may lead to organ shape asymmetry.

\section{Results}

Dynamic changes in the asymmetry of epidermal cell wall elasticity during early leaf growth. We recently found that the adaxial domain has a lower auxin concentration than the abaxial domain

\footnotetext{
'State Key Laboratory of Plant Genomics, Institute of Genetics and Developmental Biology, Chinese Academy of Sciences, and National Center for Plant Gene Research, 100101 Beijing, China. ${ }^{2}$ University of Chinese Academy of Sciences, 100049 Beijing, China. ${ }^{3}$ Key Laboratory of Microgravity (National Microgravity Laboratory), Center of Biomechanics and Bioengineering, and Beijing Key Laboratory of Engineered Construction and Mechanobiology, Institute of Mechanics, Chinese Academy of Sciences, 100190 Beijing, China. ${ }^{4}$ Bruker Nano Surfaces Business, 100081 Beijing, China. ${ }^{5}$ College of Life Sciences, Peking University, 100871 Beijing, China. Jiyan Qi, Binbin Wu and Shiliang Feng contributed equally to this work. *e-mail: mlong@imech.ac.cn; yljiao@genetics.ac.cn
} 
during early leaf development ${ }^{28}$. In the shoot, auxin can promote cell wall loosening and then cell elongation ${ }^{29,30}$, although the detailed molecular mechanism has not been fully resolved ${ }^{3}$. In this study, we first determined if mechanical heterogeneity exists between the adaxial domain and the abaxial domain. Using tomato, we applied atomic force microscopy (AFM) to directly measure the elastic modulus of the epidermal cell wall in living leaf primordia (Fig. 1 and Supplementary Figs. 1 and 2). Following a recent AFM proto$\mathrm{Col}^{5,31}$, we measured the local elastic modulus of the outer wall within different leaf domains, which is relatively independent from turgor pressure (see Methods for more details). To this end, we randomly selected multiple cells from the adaxial side facing the meristem and the opposite abaxial side of the youngest leaf primordium $\left(\mathrm{P}_{1}\right)$ (Fig. 1a). For $\mathrm{P}_{2}$ and $\mathrm{P}_{3}$ stages, we also included the middle region, the adaxial-abaxial junction (Fig. 1a). AFM allowed us to examine the morphology of the epidermal cells and the elastic modulus of their cell walls (Fig. 1b,c,e-g,i-k). We maintained indentation depth substantially lower than wall thickness, as indicated by indentation curves (Supplementary Fig. 2), to avoid artefacts. In general, cells are dome-shaped and high elastic modulus lines match the position of the anticlinal walls. Presumably, newly formed anticlinal cell walls do not lead to changes in the surface shape but result in increased elastic modulus. Our AFM-based elastic modulus map of epidermal cells revealed clear spatiotemporal mechanical differences. We quantitatively compared the elastic moduli of epidermal cell walls from regions without vertical cell walls below. At both $\mathrm{P}_{1}$ and $\mathrm{P}_{2}$ stages, we measured a higher elastic modulus from adaxial cells than abaxial cells (Fig. 1d,h and Supplementary Fig. 1c,d). At the $\mathrm{P}_{2}$ stage, cells in the middle region have elastic moduli significantly higher than cells in the abaxial side. However, the elastic moduli of the adaxial side cells decreased to a level similar to those of the abaxial side cells at the $\mathrm{P}_{3}$ stage (Fig. 11 and Supplementary Fig. 1e). The cells in the middle region continued to have high elastic moduli at the $\mathrm{P}_{3}$ stage, even higher than at the $\mathrm{P}_{2}$ stage. By using a different AFM setup and protocol with the ramp mode ${ }^{31}$, we obtained similar differences among domains (Supplementary Fig. 1a,b), as we did with the quantitative nano-mechanical (QNM) mode (Fig. 1). Taken together, our observations show that the asymmetry of cell wall mechanical properties change dynamically during early leaf development.
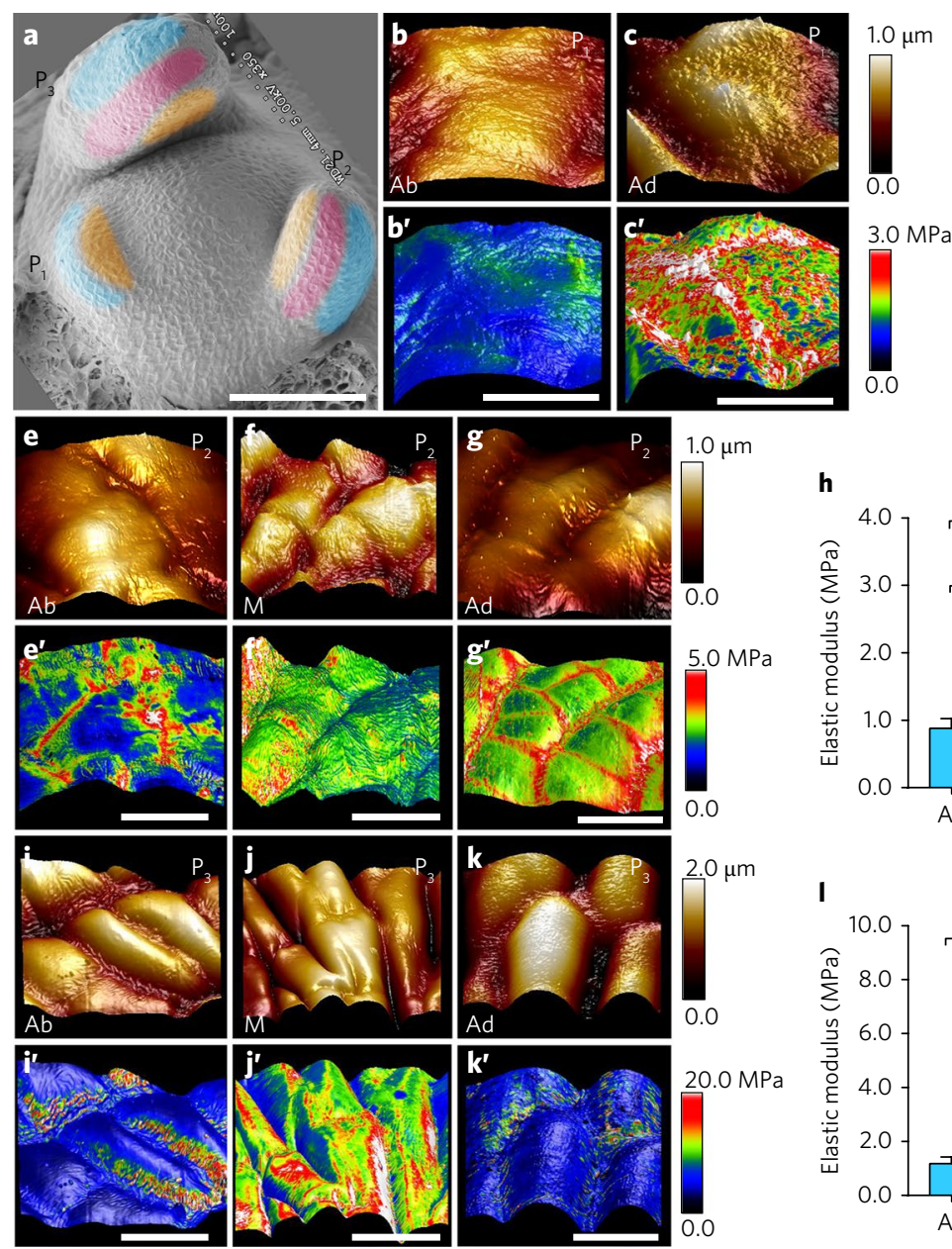

h
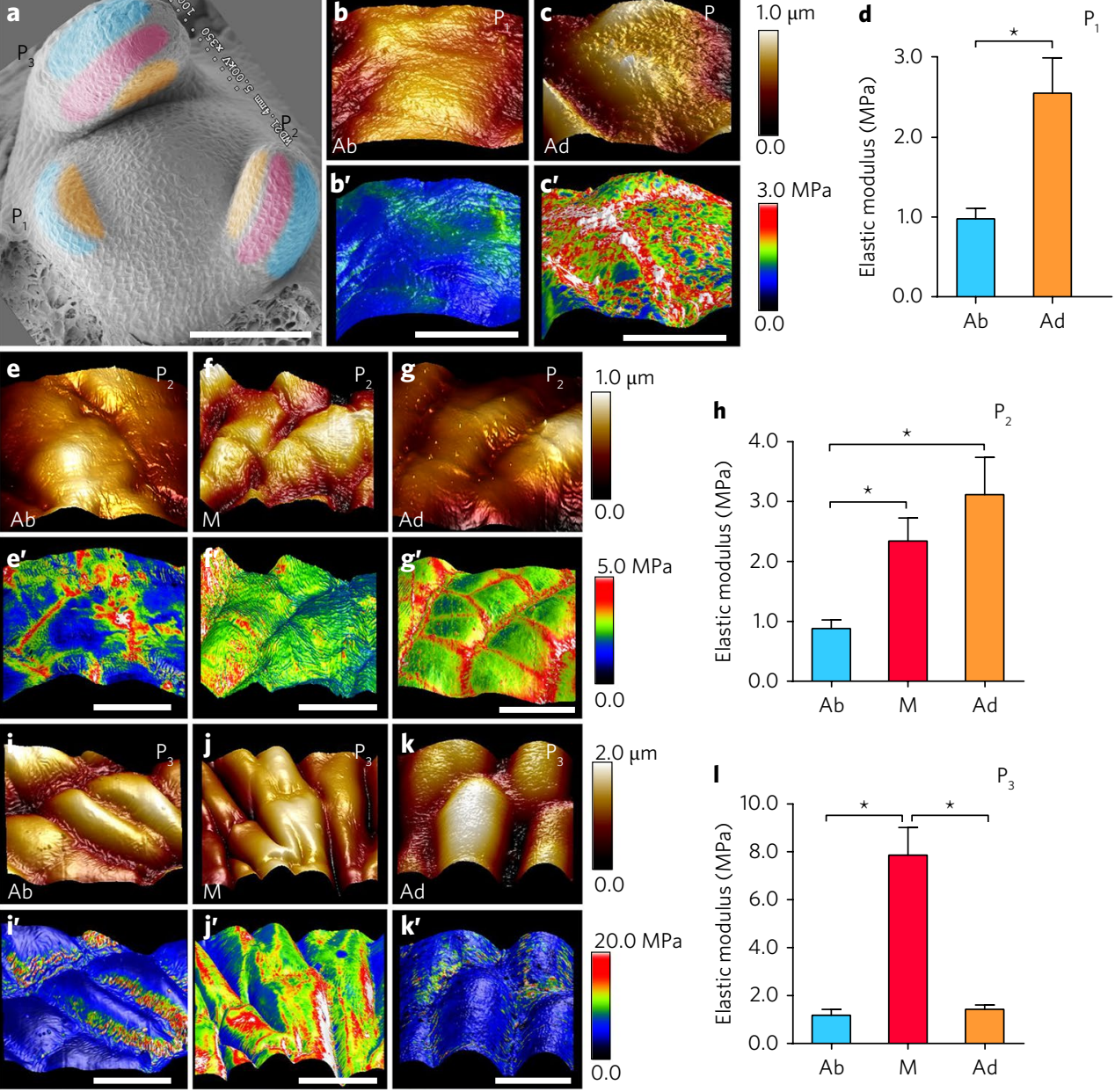

Fig. 1 | Dynamic changes of the asymmetry of the elastic modulus of epidermal cell walls. Measurement of domain-specific elastic modulus in $P_{1}-P_{3}$ stage tomato leaves. $\mathbf{a}$, Top view of a tomato shoot apex showing the adaxial side ( $A d$, orange) and abaxial side ( $A b$, blue) of $P_{1}$, $P_{2}$ and $P_{3}$, and the middle region $\left(M\right.$, red) in $P_{2}$ and $P_{3} . \mathbf{b}, \mathbf{c}, \mathbf{e}-\mathbf{g}, \mathbf{i}-\mathbf{k}$, Three-dimensional rendering of epidermal cell topography and elasticity obtained by $A F M$ using the PeakForce $Q N M$ mode, with $\mathbf{b}$ and $\mathbf{c}$ from $\mathrm{P}_{1}, \mathbf{e}-\mathbf{g}$ from $\mathrm{P}_{2}$ and $\mathbf{i}-\mathbf{k}$ from $\mathrm{P}_{3}$. Each upper panel shows a three-dimensional rendering of cell topography alone, and each lower panel presents cell topography overlaid with elasticity. Note that different scales were used for each stage, and also the differences in elastic modulus values among $\mathrm{Ad}, \mathrm{Ab}$ and $\mathrm{M}$ cells. d,h,l, Quantification of epidermal cells' apparent Young's modulus obtained by $\mathrm{AFM}$ using the QNM mode in $\mathrm{P}_{1}$ (d), $\mathrm{P}_{2}$ (h) and $\mathrm{P}_{3}(\mathbf{I})$. A total of 24 cells in six leaf samples were recorded per region at each stage, with raw AFM measurements provided in Supplementary Table 2. Values are mean \pm s.e.m. ${ }^{\star}$ Student's $t$-test $P<0.0001$ and the value change above twofold. Scale bars, $100 \mu \mathrm{m}$ in $\mathbf{a}$; and $10 \mu \mathrm{m}$ in $\mathbf{b}, \mathbf{c}, \mathbf{e}-\mathbf{g}$ and $\mathbf{i}-\mathbf{k}$. 
Three domains with different methyl-esterification status of cell wall pectins. Recent work has related de-methyl-esterification of pectins, a major component of the primary cell wall, with wall elasticity $^{14}$. In particular, pectin de-methyl-esterification may be controlled by auxin ${ }^{29}$, which has asymmetric distribution in early leaf primordia $^{28}$. Thus, we focused on pectin de-methyl-esterification. We used the monoclonal antibodies LM19 and JIM5 ${ }^{32,33}$, which specifically label de-methyl-esterified but not methyl-esterified pectin homogalacturonan (HG), to analyse the methyl-esterification status of $\mathrm{HG}$ in emerging tomato and Arabidopsis leaves. We also used the 2F4 antibody, which detects $\mathrm{Ca}^{2+}$-crosslinked $\mathrm{HG}$ with up to $40 \%$ methyl-esterification $^{34}$ and is commonly used to recognize low methyl-esterified $\mathrm{HG}^{35}$. In the tomato shoot apex, we labelled successive transverse sections through the SAM and leaf primordia with 2F4 (Fig. 2a-d and Supplementary Fig. 3a-c), LM19 (Supplementary Fig. 4a-c) or JIM5 (Supplementary Fig. 4e-g). The centre of the SAM was unlabelled, as described previously for Arabidopsis $^{15}$, but leaf primordia showed dynamic, domain-specific labelling. The labelling patterns of 2F4, LM19 and JIM5 were generally consistent with each other. The small size of early $\mathrm{P}_{1}$ stage leaves prohibited us from unambiguously detecting signals (Fig. 2 and Supplementary Fig. $3 \mathrm{~d}-\mathrm{e}, \mathrm{g}-\mathrm{h})$. In $\mathrm{P}_{2}$ and older stage leaves, we observed striking labelling in the walls of abaxial domain cells, suggesting selective $\mathrm{HG}$ de-methyl-esterification in this region (Fig. 2 and Supplementary Figs. $3 \mathrm{a}-\mathrm{c}$ and $4 \mathrm{a}-\mathrm{c}, \mathrm{e}-\mathrm{g}$ ). Starting from the $\mathrm{P}_{3}$ stage, cell walls in the adaxial domain were also labelled (Fig. 2 and Supplementary Fig. $4 \mathrm{a}-\mathrm{c}, \mathrm{e}-\mathrm{g}, \mathrm{i}$ ), but the middle domain remained unlabelled until the $P_{5}$ stage. The outermost layer of epidermal cell walls had much weaker labelling than inner cell walls (Supplementary Fig. 3i), suggesting higher HG methyl-esterification in the outermost cell walls. In chemically de-esterified controls, we observed strong homogenous labelling of all cell walls in the shoot apex, including leaves (Supplementary Figs. 3l-n and 4d,h,l). We also detected partially methyl-esterified HG with LM20, which does not bind to unesterified HG, and obtained a complementary staining pattern to that of 2F4, LM19 or JIM5 (Supplementary Fig. 4j). No signal was detected when no primary antibody was added (Supplementary Fig. 4m,n), excluding non-specific crossreactivity of the secondary antibody. In addition to tomato, we found similar labelling patterns in Arabidopsis leaves (Fig. 2e-h and Supplementary Fig. 3f-h), suggesting evolutionary conservation of the observed HG methyl-esterification dynamics.

We next confirmed that cell wall pectin de-methyl-esterification has significant effects on wall elasticity in leaf primordia. In tomato, we locally applied pectin methylesterase (PME), which is responsible for in vivo de-methyl-esterification of pectin. We positioned one or more sepharose beads loaded with citrus PME on the adaxial side of tomato $\mathrm{P}_{1}$ leaf primordia under the microscope (Supplementary Fig. 5a), which resulted in ectopic HG de-methylesterification in the adaxial domain (Supplementary Fig. 5b,d). Furthermore, the PME treatment substantially reduced the elastic moduli of the treated side (Supplementary Figs. 4o and 5f-h). We also locally applied lanolin containing the catechin-derivative (-)-epigallocatechin gallate (EGCG), an inhibitor of PME activity ${ }^{13}$, to the abaxial domain of tomato $\mathrm{P}_{1}$ leaf primordia. Similarly, the EGCG treatment ectopically reduced HG de-methyl-esterification (Supplementary Fig. 5b,c) and increased the elastic moduli of the abaxial side (Supplementary Figs. 4o and 5e,g-h). Thus, we concluded that cell wall pectin de-methyl-esterification negatively correlates with wall elasticity in the epidermis in leaf primordia, as in the shoot apex ${ }^{14}$. However, outermost cell walls of epidermal cells are uniformly methyl-esterified (Supplementary Fig. 3i), suggesting an additional regulatory mechanism of wall elastic modulus. Nevertheless, inner cells with higher wall de-methylesterification and epidermal cells with lower elastic moduli are located in the same domain. Similarly, inner cells with lower wall
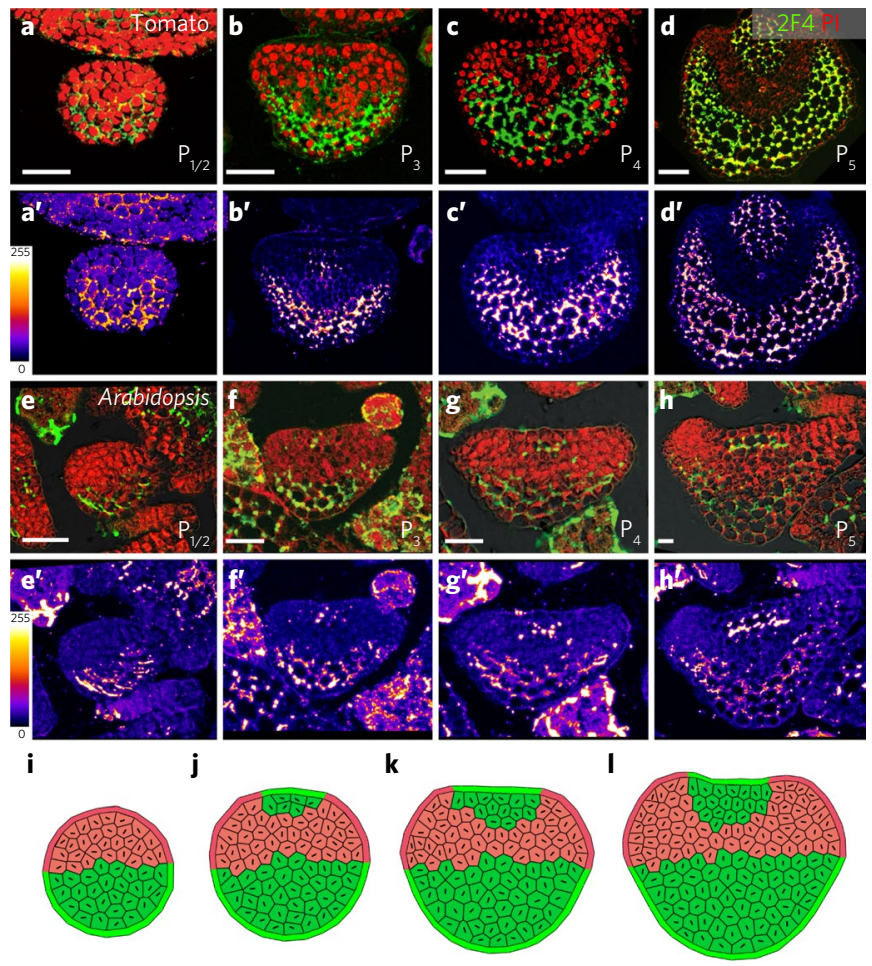

Fig. 2 | Methyl-esterification of cell wall pectin in leaf primordia. Transverse sections through tomato (a-d) and Arabidopsis (e-h) leaf primordia stained with propidium iodide (red) showing 2F4 labelling (green) of low HG methyl-esterification. Each upper panel shows a confocal image, and each lower panel is a heat map. More examples are shown in Supplementary Figs. 3 and 4. i-I, Growth simulation of multicellular leaf transverse sections. The leftmost shape represents the initial round shape with domain-specific rigidities. The subsequent steps are shown to the right. Green represents low rigidity and red represents high rigidity. Scale bars, $20 \mu \mathrm{m}$. 2F4, monoclonal antibody to homogalacturonan, in green. PI, propidium iodide, in red.

de-methyl-esterification and epidermal cells with higher elastic moduli are located in the same domain.

To test if the chemical and genetic signals affecting leaf polarity regulate HG methyl-esterification, we first manipulated auxin distribution and signalling in tomato leaf primordia. We applied lanolin containing auxin (indole-3-acetic acid) to the adaxial side of tomato $\mathrm{P}_{1}$, which would cause radial leaves ${ }^{28}$. As shown in the shoot apex ${ }^{29}$, HG de-methyl-esterification increased after auxin treatment (Supplementary Fig. 6b). By AFM, we also confirmed that auxin treatment decreased the elastic moduli of the adaxial side (Supplementary Fig. 6d-f). We next abaxially applied the auxin antagonistic auxinole, which binds TIR1/AFB proteins to block auxin signalling ${ }^{36}$. We found that this treatment could also induce radial leaves (34 out of 104). Auxinole treatment led to reduced HG de-methyl-esterification in the abaxial domain (Supplementary Fig. 6a) and increased elastic moduli (Supplementary Figs. 40 and 6c,e-f).

To determine whether adaxial-abaxial leaf polarity influences pectin methyl-esterification, we next analysed the HG methylesterification status in Arabidopsis lines with leaf polarity defects. The $p M P:: M P \Delta$ plants with ectopic auxin signalling in the adaxial domain have abaxialized leaves ${ }^{28,37}$. We hypothesized that $p M P:: M P \Delta$ leaves may acquire precocious HG de-methyl-esterification in the adaxial side. Indeed, we detected high HG de-methyl-esterification in both the adaxial and abaxial domains, but not the middle domain of young leaf primordia (Supplementary Fig. 3j). We also speculated that the phavoluta-1d $(p h v-1 d)$ mutant with fully adaxialized 
leaves $^{38}$ might have delayed HG de-methyl-esterification. In leaf primordia until the $\mathrm{P}_{7}$ stage, we could not detect HG de-methylesterification (Supplementary Fig. 3k). Based on previous reports in Arabidopsis ${ }^{6}$ and observations in tomato, we expect correlated wall elasticity changes with pectin methyl-esterification status. Taken together, our results suggest that developmental regulators of leaf polarity also affect pectin methyl-esterification status, and thus wall elasticity.

Asymmetric cell wall elasticity is sufficient for asymmetric leaf growth. Previous theoretical predictions suggest that early patterns of growth can generate organ asymmetry in animals and plant ${ }^{39-42}$. Changes in elasticity correlates with wall extensibility can lead to differences in cell expansion and growth ${ }^{10,11}$. Thus, we next used a computational model to predict whether the observed dynamics of mechanical properties are sufficient to generate shape change and to produce asymmetric leaves.

To determine how dynamic differences in mechanical properties can generate asymmetry in leaves, we implemented a conceptual modelling framework that can describe the epidermal tissue and the inner tissue. We developed a two-dimensional (2D) hybrid model specialized for regional differences in mechanical properties, which also avoids the complexity of cellularized growth (Supplementary Fig. 7a,b). Briefly, this model considers the epidermis as a continuous, viscoelastic material without explicitly individualized epidermal cells and with non-cell autonomous expansion, which we used the finite element method to describe ${ }^{43}$. The inner cells follow the widely accepted concept of turgor-driven plant cell growth, in which cell expansion involves irreversible yielding of the cell walls to high internal turgor pressure ${ }^{10,11}$. We used the energy minimizing method to describe inner cells ${ }^{44}$. The generalized Maxwell solid model (Supplementary Fig. 7) for mimicking the epidermis contains three parallel elements: two Maxwell elements (accounting for the viscoelastic effect) and one spring element (accounting for the long-term or steady-state elastic response). More details of the model and the strategies bridging the gap between AFM measurement and numerical simulation are described in the Methods and in the Supplementary Information.

To test the importance of cell wall mechanical dynamics on asymmetric leaf growth, we applied the above model to a $2 \mathrm{D}$ template, as a proxy for a leaf cross-section. Starting from a round shape, the morphological evolution of the modelled leaf is governed by the energy minimization principle for the inner cells, coordinated with mechanical balance for the epidermis upon various mechanical parameter settings of both inner and epidermal cell walls. Assuming increased pectin de-methyl-esterification increases elasticity ${ }^{6,15}$, the observed inner cell pectin methyl-esterification status and neighbouring epidermal cell elasticity share similar trends-that is, more elastic epidermal cells cover de-methyl-esterified inner cells. Like inner cells, mechanical properties of the epidermis are differentially regulated among abaxial, middle and adaxial regions. We assumed a link between the wall elasticity and cell growth, which is further associated with wall plasticity ${ }^{45}$. In the model, we allowed local softening of inner cell wall in accord with that of the neighbouring epidermis. In addition, we applied an epidermal restriction-that is, higher wall elastic modulus than neighbouring inner cellswhich we describe below. To mimic the softening behaviour in our model setting, as observed experimentally, we allowed a certain number of inner cells and their adjacent epidermal cells to change their properties when the area of leaf surpassed a threshold value. Such dynamically changing wall mechanics resulted in formation of organ asymmetry that mimics the shape of a $\mathrm{P}_{3 / 4}$ leaf (Fig. 2i-1, Supplementary Fig. 7a and Supplementary Video 1). Therefore, differences in cell wall mechanical properties alone are sufficient for the formation of organ asymmetry in the model. The exact shape and extent of asymmetry depended on model parameters such as

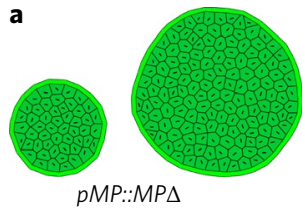

b
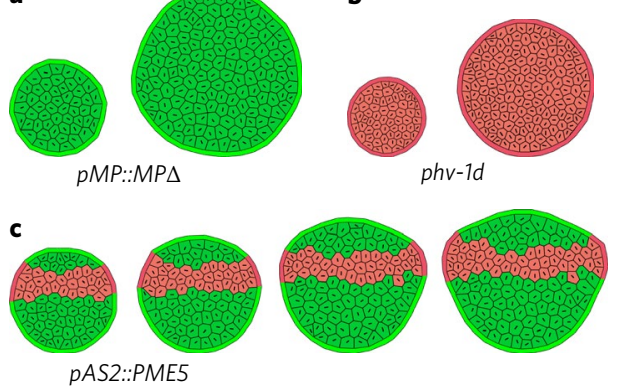

西
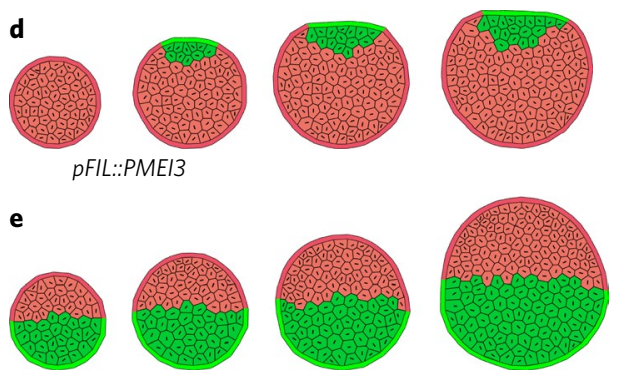

Fig. 3 | A conceptual mechanical model is sufficient to predict organ asymmetry formation. Growth simulation of multi-cellular leaf transverse sections. a, Reduced cell wall elasticity in the adaxial and middle domain. b, Increased cell wall elasticity in the abaxial and adaxial domain. c, Hastened adaxial cell wall loosening, in which the adaxial and abaxial domains have identical wall elasticity patterns. Asymmetry is reduced.

d, Two-domain partition with the abaxial domain acquiring the middle domain cell wall property, that is, constitutively stiffening in the abaxial and middle domains. e, An alternative two-domain partition with the adaxial domain acquiring the middle domain cell wall property. In all the simulations, the leftmost shape represents the initial round shape with domain-specific rigidities. The subsequent steps are shown to the right. Panels $\mathbf{a}$ and $\mathbf{b}$ show only the initial step and the final step. Green represents low rigidity and red represents high rigidity. The perturbations in the model are labelled with approximately corresponding Arabidopsis genotypes.

domain partition, but the formation of asymmetry was a robust feature within a wide range of mechanical parameters (Supplementary Fig. 7c). From a physical perspective, the stiff cells receive stronger constraints from their neighbouring epidermal cells, such that they prefer to grow and divide by pressing on the soft inner cells, and vice versa for the soft cells. The final shape of the leaf is the manifestation of the actual growth rate of different groups of inner cells, which is determined by the physical constraints within a multicellular system (Supplementary Fig. 7d).

We next varied the dynamic patterns of wall elasticity. We first assigned the abaxial domain wall elasticity pattern to the adaxial and middle domains, which is similar to $p M P:: M P \Delta$ plants, although there are remaining presumably low wall elasticity inner cells in pMP::MPA leaves. This created an enlarged, radially symmetrical shape (Fig. 3a). We similarly assigned the adaxial domain wall elasticity pattern to the abaxial domain, as seen in $p h v-1 d$ plants. Again, this created an enlarged, radially symmetrical shape (Fig. 3b). We also asked if the observed temporal wall elasticity pattern changes are important for morphogenesis. To this end, we initiated adaxial domain wall loosening earlier in the model, and obtained reduced symmetry (Fig. 3c and Supplementary Video 2). In addition, we consolidated three domains into two domains. Combining the middle domain with either the abaxial domain or the adaxial domain failed to recapitulate normal establishment of asymmetric leaf shape (Fig. 3d,e and Supplementary Video 3). Given the model assumptions, these results suggest that asymmetric organ growth requires the observed mechanical properties and cell wall dynamics. 
Establishment of leaf asymmetry requires cell wall mechanical property dynamics. To specifically alter cell wall mechanical properties and test model predictions, we used exogenous enzymes and chemicals to alter pectin methyl-esterification status in specific leaf domains. We first locally applied the treatments in tomato. We used adaxial-specific PME treatment to decrease the cell wall elastic modulus in the adaxial (and possibly middle) domains (Fig. 3a,c). Consistent with the prediction of the model, we reproducibly observed partial or full loss of leaf asymmetry in treated leaves (60 out of 132, Fig. 4a-d). Epidermal morphology and vascular tissue structure suggested conversion of adaxial identity to abaxial identity (Fig. 4d). Non-glandular trichomes (Supplementary Fig. 8h), which are typical to abaxial epidermis ${ }^{28}$, dominated the adaxial epidermis. Next, we used abaxial-specific EGCG treatment to increase cell wall elastic modulus in the abaxial (and possibly middle) domains (Fig. 3b,d). We obtained leaves with a range of polarity defects (13 out of 128), including radially symmetrical, split or disappeared vascular tissue (Fig. 4e,f and Supplementary Fig. 8a-c). On the epidermis, abaxial-specific EGCG treatment also led to mostly abaxial non-glandular trichomes (Supplementary Fig. 8g).
Furthermore, we simultaneously applied PME to the adaxial side and EGCG to the abaxial side of $\mathrm{P}_{1}$. Again, this double treatment resulted in abaxialized leaves rather than flipped adaxial-abaxial polarity (26 out of 46), as suggested by non-glandular trichomes (Supplementary Fig. 8i,j). Treatment of leaf primordia older than $\mathrm{P}_{1}$ no longer affected morphological asymmetry, suggesting that the mechanical signal functions early in leaf development. In our control experiments, in which leaf primordia were treated with PME on the abaxial side (none out of 51), EGCG adaxially (none out of 53) or adaxial treatment with denatured PME (none out of 37), we did not observe any radially symmetrical leaves. Thus, stress induced by PME or EGCG treatment, if there is any, is insufficient to cause polarity growth defects.

We also tested the model predictions in Arabidopsis using transgenic plants. The Arabidopsis genome encodes PMEs and endogenous PME inhibitor (PMEI) proteins ${ }^{46}$. Both families are highly redundant, with 66 members in the PME family and 69 members in the PMEI family ${ }^{15}$; moreover, many PME and PMEI genes are expressed in leaves ${ }^{47}$, hindering mutant analysis. We therefore adopted a domain-specific ectopic-expression approach.
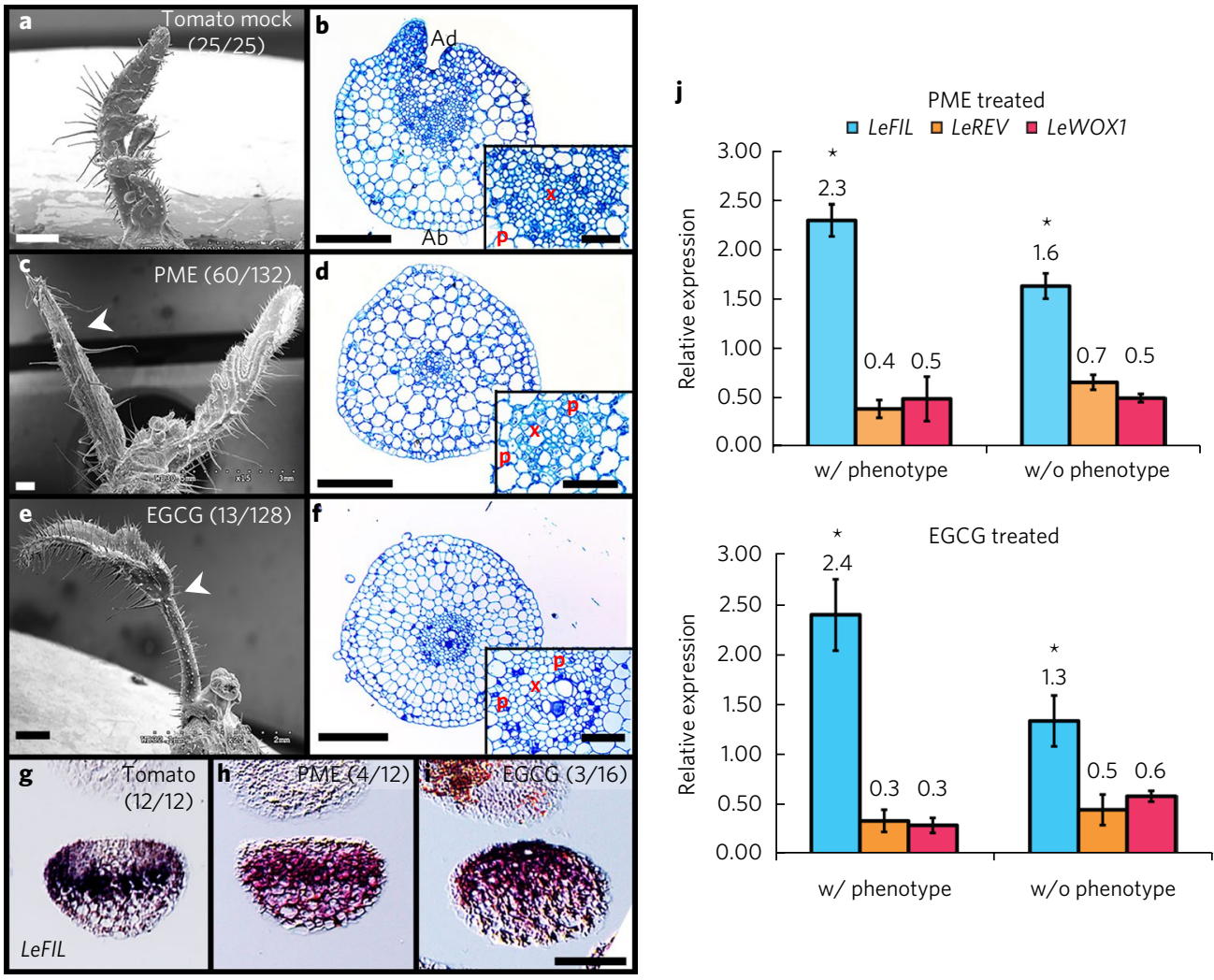

Fig. 4 | Dynamics of cell wall pectin methyl-esterification are critical for leaf polarity patterning in tomato. $\mathbf{a}, \mathbf{b}$, Control tomato leaf primordia, showing a primordium 7 days after emergence (a) and a transverse section through the midrib and adjacent lamina regions with close-up insertion of vascular strand (b). c,d, Tomato leaf primordia after adaxial microapplication of PME beads, showing an early leaf (c) and a transverse section through the midrib and adjacent lamina regions (d). Note that phloem cells $(p)$ surround the xylem ( $x$ ) elements. e,f, Tomato leaf primordia after abaxial microapplication of EGCG-containing lanolin, showing an early leaf (e) and a transverse section through the midrib and adjacent laminal regions (f). More examples are shown in Supplementary Fig. 9. g-i, Patterns of LeFIL transcript accumulation in transverse sections through untreated (g), PME-treated (h) and EGCGtreated (i) $P_{1}$. Samples were fixed $48 \mathrm{hr}$ after treatment and all leaves have the adaxial side facing up. LeFIL accumulation in the untreated leaf is excluded from the adaxial domain $(\mathbf{g})$, but is invaded or even enriched in the adaxial domain after treatment ( $\mathbf{h}$ and $\mathbf{i})$. Fractions of samples showing the displayed features are in parentheses. Note that mis-expression may be underestimated due to difficulties in unambiguously scoring images. $\mathbf{j}$, Relative expression of selected polarity genes in mock-, adaxial PME- and abaxial EGCG-treated tomato leaf primordia. RNA was isolated from stage $\mathrm{P}_{4 / 5}$ leaf primordia with and without detectable morphological changes. Transcript levels were measured by RT-qPCR in triplicate. Results in treated leaf primordia were normalized against expression in mock-treated leaf primordia of the same experimental pair. Values are mean \pm s.d., with three or more biological replicates, and five leaves were used for each biological replicate. *Student's $t$-test $P<0.01$. Scale bars, $1 \mathrm{~mm}$ in $\mathbf{a}, \mathbf{c}$ and $\mathbf{e} ; 100 \mu \mathrm{m}$ in $\mathbf{b}, \mathbf{d}$ and $\mathbf{f}$; and $50 \mu \mathrm{m}$ in $\mathbf{i}$ and in insertions of $\mathbf{b}, \mathbf{d}$ and $\mathbf{f}$. w/, with detectable morphological changes; w/o, without detectable morphological changes. 
To this end, we expressed Arabidopsis PME5 under the adaxially expressed ASYMMETRIC LEAVES2 (AS2) promoter, and expressed Arabidopsis PMEI3 under the abaxially expressed FILAMENTOUS FLOWER (FIL) promoter. We introduced these transgenes into Col-0 wild-type plants and the revoluta-6 (rev-6) background, which does not have leaf adaxial-abaxial polarity defects but is more sensitive to alterations of leaf patterning (Fig. $5 \mathrm{a}-\mathrm{h})^{48}$. The domain-specific AS2 and FIL promoter activities were not changed in the mutant background (Supplementary Fig. 9a-d). Although the AS2 promoter is activated mostly in the adaxial epidermis (Supplementary Fig. 9a and b), we could still detect polarity defects in 27 of 75 independent rev-6 lines transgenic for pAS2::PME5 (Fig. 5e,f). We also obtained lines with radially symmetrical leaves in eight of 24 independent rev-6 lines transgenic for pFIL::PMEI3, which ectopically express PMEI3 in the abaxial and middle domains ${ }^{49}$ (Supplementary Fig. 9c-e). Similar results were obtained in the Col-0 wild type, as in rev-6 (Supplementary Fig. 9h,i and Supplementary Table 1). Vascular tissue structure and epidermal morphology of symmetric leaves from both genotypes suggested abaxialization (Fig. 5f,h and Supplementary Fig. 10a-f). However, we were unable to obtain transgenic lines with high-level transgene expression, and the obtained transgenic lines were often infertile, indicating that transformants with strong expression could be embryonic or early seedling lethal. Taken together, these experimental results validate the model predictions that cell wall mechanical property dynamics lead to leaf asymmetry (Fig. 3c,d).

In addition to leaf morphology changes, we observed gene expression changes in plants with altered pectin methyl-esterification status. In situ analysis uncovered an adaxialized and enlarged domain of LeFIL expression in adaxial-specific PME-treated and abaxial-specific EGCG-treated tomato leaf primordia (Fig. 4g-i). Whereas LeFIL expression is restricted to the abaxial and middle domains in normal leaves ${ }^{49}$, it was detected in the adaxial region after treatment. In Arabidopsis, FIL expression was similarly enlarged in young $p A S 2:: P M E 5$ rev-6, but not rev-6, leaf primordia (Supplementary Fig. 9c-e). By contrast, the adaxially expressed LeREV had reduced and less defined expression after either PME or EGCG treatment (Supplementary Fig. 8d-f). We further used quantitative PCR with reverse transcription (RT-qPCR) analysis to quantify gene expression in mock-, PME- or EGCG-treated leaf primordia. Compared with mock-treated leaf primordia, the expression of LeFIL increased substantially in both adaxial PMEtreated and abaxial EGCG-treated tomato $\mathrm{P}_{4 / 5}$ stage leaf primordia (Fig. 4j). By contrast, the expression of LeREV and the middle domain gene LeWOX1 significantly decreased. Thus, mechanical signals may feed back on gene expression. This feedback could be direct through the mechanical signals or indirect through chemical signals such as oligo galacturonic acids. Note that both hypotheses are not mutually exclusive.

Epidermal restriction is required for asymmetric leaf growth. In addition to cell wall elasticity, our modelling simulation predicted that epidermal restriction is necessary for asymmetric leaf growth. Removal of epidermal restriction by assigning epidermal cell walls a uniform, low elastic modulus led to reduced leaf shape symmetry (Fig. 6a and Supplementary Video 4). Assigning the epidermis a uniform, high elastic modulus in the slow creeping phase also led to a less symmetrical shape (Fig. 6b and Supplementary Video 5). Although domain-specific epidermal restriction is necessary for asymmetric leaf growth, it is not sufficient with uniform inner cell wall elasticity (Fig. 6c).

We experimentally tested these predictions from the modelling simulation in Arabidopsis. To this end, we expressed either $P M E 5$ or $P M E I 3$ under the epidermal layer-specific ARABIDOPSIS THALIANA MERISTEM LAYER1 (ATML1) promoter $^{50}$ in wild-type and as2-2 plants (Supplementary Fig. 9f,g), and obtained similar leaf

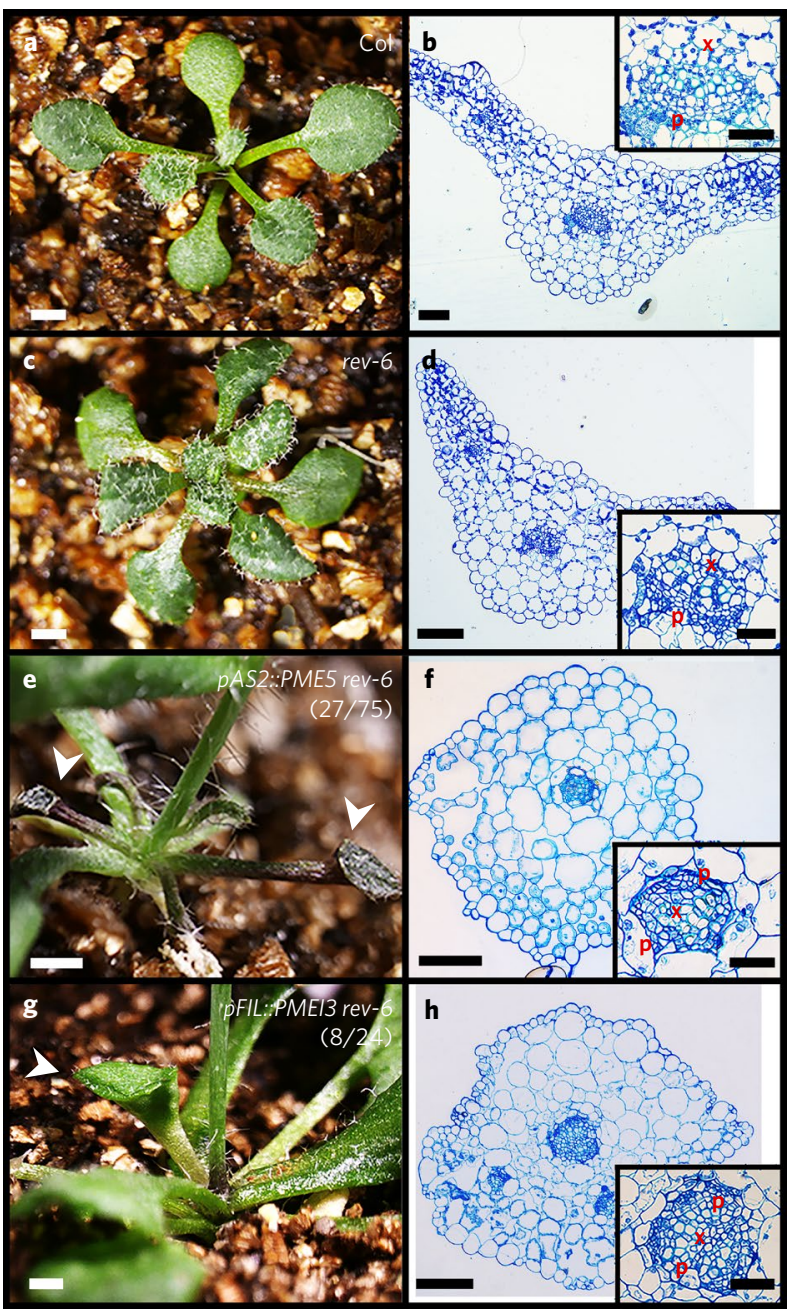

Fig. 5 | Dynamics of cell wall pectin methyl-esterification are critical for leaf polarity patterning in Arabidopsis. Arabidopsis Col-O wild-type (a), rev-6 (c), pAS2::PME5 rev-6 (e) and pFIL::PMEI3 rev-6 (g) plants showing leaf morphology. Arrowheads highlight leaves with disrupted polarity in $\mathbf{e}$ and $\mathbf{g}$. b,d,f,h, Transverse sections through blade base regions of Col-0 wild-type (b), rev-6 (d), pAS2::PME5 rev-6 (f) and pFIL::PMEI3 rev-6 (h) leaves. Mesophyll morphology and vascular structure indicate abaxialization in transgenic plants. Fractions of samples showing the displayed features are in parentheses. Scale bars, $1 \mathrm{~mm}$ in $\mathbf{a}, \mathbf{c}$, e and $\mathbf{g}$; $100 \mu \mathrm{m}$ in $\mathbf{b}, \mathbf{d}, \mathbf{f}$ and $\mathbf{h}$; and $50 \mu \mathrm{m}$ in insertions of $\mathbf{b}, \mathbf{d}, \mathbf{f}$ and $\mathbf{h}$. Col, Col-0 wild-type; $p$, phloem cells; $x$, xylem cells.

polarity defects. Within the epidermis, we observed that enhanced HG de-methyl-esterification increased in pATML1::PME5 plants. In $p A T M L 1:: P M E I 3$ plants, the walls in between L1 and L2 no longer had HG de-methyl-esterification (Supplementary Fig. 11). The as 2-2 mutant does not exhibit obvious adaxial defects, but is more sensitive to alterations of leaf patterning. In five out of 27 transgenic T1 pATML1::PME5 as2-2 plants (and their progenies if available), we identified trumpet-like or rod-like symmetric leaves. Analysis of vascular tissue structure and epidermal morphology indicated these abnormal leaves were abaxialized (Fig. 6d-g). Similarly, we identified abaxialized leaves in five out of 24 transgenic T1 pATML1::PMEI3 as2-2 plants (Fig. 6h,i). Similar results were obtained in the Col-0 wild type (Supplementary Fig. 9j,k and Supplementary Table 1). These experiments validated the importance of epidermal restriction, as predicted by our model. 


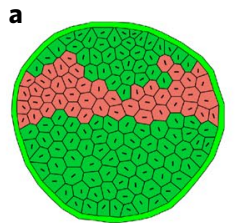

PATML1::PME5 b

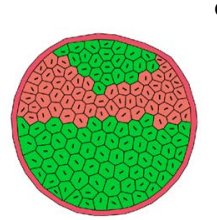

PATML1::PMEI3

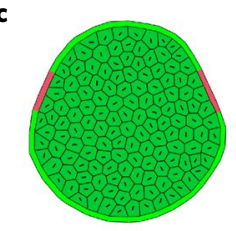

as2-2 $\mathbf{e}$

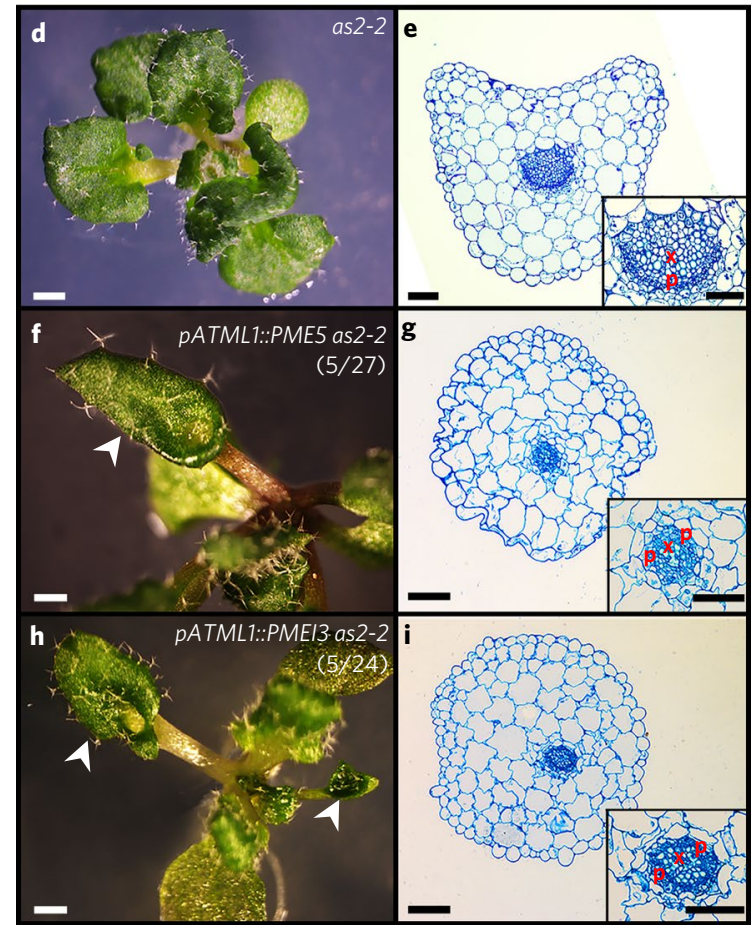

j

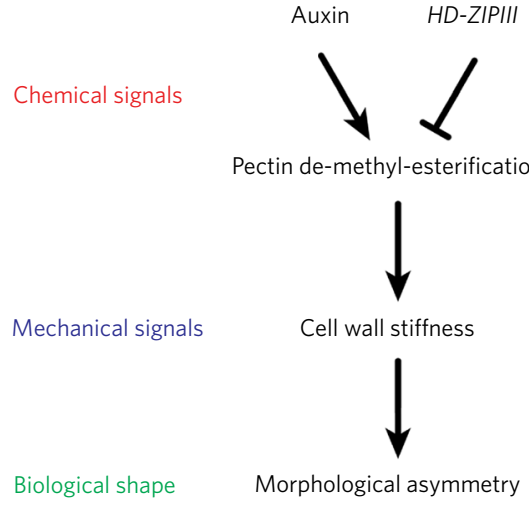

Fig. 6 | Epidermal restriction is necessary for polarity patterning. a-c, Final steps obtained in growth simulation of multi-cellular leaf transverse sections, as seen in Figs. 2 and 3. Green represents low rigidity and red represents high rigidity. Virtual leaf cross-sections with uniformly reduced epidermal restriction (a), with uniformly enhanced epidermal restriction (b) or with uniform inner cell wall elasticity (c). Endpoints of simulations show lack of polarity establishment. d,f,h, Arabidopsis as2-2 (d), pATML1::PME5 as2-2 (f) and pATML1::PMEI3 as2-2 (h) plants showing leaf morphology. Arrowheads highlight leaves with disrupted polarity in $\mathbf{f}$ and $\mathbf{h} . \mathbf{e}, \mathbf{g}, \mathbf{i}$, Transverse sections through petiole regions of as2-2 (e), pATML1::PME5 as2-2 (g) and pATML1::PMEI3 as2-2 (i) leaves. Mesophyll morphology and vascular structure indicate abaxialization in transgenic plants. j, Polarity genes and auxin regulate leaf polarity patterning through mechanical signals. Fractions of samples showing the displayed features are in parentheses. Scale bars, $1 \mathrm{~mm}$ in $\mathbf{d}, \mathbf{f}$, and $\mathbf{h} ; 100 \mu \mathrm{m}$ in $\mathbf{e}, \mathbf{g}$ and $\mathbf{i}$; and $50 \mu \mathrm{m}$ in insertions in $\mathbf{e}, \mathbf{g}$ and $\mathbf{i}$. p, phloem cells; $x$, xylem cells.

\section{Discussion}

Although molecular genetic studies have identified and deciphered many of the signalling pathways that regulate patterning, the mechanisms that generate dynamic yet precise biological shapes during organ growth remain an open area of investigation ${ }^{51}$. Mechanical influences on development have long been proposed, and recent studies in plants and animals have started to reveal the importance of mechanical factors. Recent studies in animal cells have proposed that tissue-level mechanical stress is needed to explain a range of developmental phenomena ${ }^{2,52}$. We previously identified abaxialenriched auxin distribution in early leaf primordia ${ }^{28}$, which may lead to domain-specific cell wall loosening ${ }^{29,30}$. In this study, we found that relatively simple changes in mechanical properties can account for dynamic shape changes during asymmetric leaf development (Fig. 6j), providing a simple unifying framework for the control of asymmetric development of organs. By connecting gene expression with wall modifier activity and wall elasticity, we were able to link gene activity to leaf adaxial-abaxial growth asymmetry. We found that pectin methyl-esterification status of inner cell walls is likely causal to inner cell wall elasticity difference. Outermost cell walls of epidermal cells presumably share similar trends of elastic moduli with neighbouring inner cells, although a pectin-independent regulation is likely involved. A recent study showed that wall mechanics underlie sepal proximo-distal organ polarity growth ${ }^{35}$, which is achieved through feedback of mechanical forces on microtubule array organization. Whether microtubule re-orientation affects leaf adaxial-abaxial asymmetry remains an open question. Mechanical signals may also affect the expression of genes affecting leaf polarity. In addition, monocot cell walls are very low in pectins, suggesting the involvement of an additional biochemical process in monocots. The importance of epidermal restriction has long been discussed and debated ${ }^{53}$, and our experimental and theoretical studies support the idea that the epidermis plays a leading role in restricting and controlling growth (Fig. 6d-i).

Our analysis of the methyl-esterification status of cell-wall pectins identified three distinct, non-overlapping domains. These domains show a temporal sequence of de-methyl-esterification, with the abaxial domain first, then the adaxial and finally the middle domain (Fig. 2). These patterns support the existence of the recently proposed middle domain, which expresses WUSCHELRELATED HOMEOBOX genes ${ }^{49}$. Our modelling simulation shows that the middle domain is necessary for leaf asymmetry and flattening (Fig. 3d,e). The dynamic changes of wall mechanics also indicated that there is no simple association between wall elasticity and polarity gene expression, such as adaxial genes associated with stiffened walls. It is likely that the absolute elasticity of the cells is less important than the differences among the three domains, as our model simulation suggests. Without a mechanical difference along the adaxial-abaxial axis, the abaxial fate will be the default fate of leaf cells ${ }^{22}$.

\section{Methods}

Plant materials and growth conditions. Tomato (Solanum lycopersicum) cultivar Castlemart plants were grown in MS medium under controlled conditions $\left(25^{\circ} \mathrm{C}\right.$ at $60 \%$ humidity, with $16 \mathrm{hr}$ of light and $22^{\circ} \mathrm{C} 8 \mathrm{hr}$ of dark) until the fifth to seventh plastochron stage. Shoot apices were dissected, leaving only $\mathrm{P}_{1}$ or $\mathrm{P}_{1}$ and $\mathrm{P}_{2}$ intact, together with the subapical region, and cultured on MS medium containing $1 \mu \mathrm{g}$ per $\mathrm{ml} t$-zeatin (PhytoTechnology Laboratories, Lenexa, KS, United States), $100 \mu \mathrm{g}$ per $\mathrm{ml}$ myo-insitol (Sigma-Aldrich, St. Louis, MO, United States) and $0.5 \mu \mathrm{g}$ per $\mathrm{ml}$ folic acid (Sigma-Aldrich) at $25^{\circ} \mathrm{C}$, with $16 \mathrm{hr}$ of light and $8 \mathrm{hr}$ of dark $^{54}$. Arabidopsis thaliana ecotypes Ler and Col-0 were used. The phv-1d mutant is in the Ler background ${ }^{55}$; the as $2-2$ and rev-6 mutants and the $p M P:: M P \Delta$ lines are in the Col-0 background ${ }^{28,56}$. Seeds were sown in pots or in plates with MS medium. Plants were incubated at $22^{\circ} \mathrm{C}$ with $16 \mathrm{hr}$ of light and $8 \mathrm{hr}$ of dark.

Confocal, optical and electron microscopy. For confocal microscopy, images were taken with a Nikon $\mathrm{Al}^{+}$confocal microscope. Excitation and detection windows for 4',6-diamidino-2-phenylindole, green fluorescent protein, propidium iodide, Alexa Fluor 488 and Alexa Fluor 546 were as previously described ${ }^{57,58}$. 
For optical microscopy, leaves were fixed in fresh formalin-acetic acid-alcohol solution under vacuum for 15-20 min, dehydrated in an ethanol series to $100 \%$ and embedded using the SPI low viscosity Spurr's kit (SPI Supplies, West Chester, $\mathrm{PA}$, United States). Sections were cut at $2 \mu \mathrm{m}$ with Leica rotary microtomes, mounted on slides and stained with $0.1 \%$ toluidine blue. Photographs were taken with a Nikon SMZ1000 stereoscopic microscope or an Olympus BX60 microscope equipped with a Nikon DS-Ril camera head.

Scanning electron microscopy was performed using a Hitachi S-3000N variable pressure scanning electron microscope after standard tissue preparation as previously described ${ }^{28,58}$. For transmission electron microscopy, Arabidopsis shoot apexes were fixed in $5 \%$ glutaraldehyde in $0.1 \mathrm{M}$ phosphate buffer $(\mathrm{pH} 7.4)$ for $4 \mathrm{hr}$ at room temperature, and then overnight in $2 \%$ osmium tetroxide at $4{ }^{\circ} \mathrm{C}$. Following three washes in phosphate buffer and distilled water, samples were stained in $1 \%$ uranyl acetate for $1 \mathrm{hr}$. Samples were washed again in distilled water and then dehydrated through a graded alcohol series and embedded in Spurr's resin (SPI Supplies). Ultrathin sections $(70 \mathrm{~nm})$ were cut using an ultramicrotome (UC7; Leica Microsystems, Wetzlar, Germany), mounted on copper grids with a single slot and stained with uranyl acetate and lead citrate. Then the samples were observed and imaged with an FEI Tecnai $\mathrm{G}^{2} 20$ TWIN electron microscope at $120 \mathrm{kV}$.

AFM. Topographical images were scanned with a Dimension Icon atomic force microscope (Bruker, Billerica, MA, United States) using the PeakForce QNM mode, which also detected for force curves. The topology image size was $20 \times 20 \mu \mathrm{m}^{2}$ or $10 \times 10 \mu \mathrm{m}^{2}$ with a resolution of $256 \times 256$ pixels. A $0.5 \mathrm{~Hz}$ scanning rate was used and the Possion's rate was set as 0.5 (ref. ${ }^{5}$ ). In addition, AFM force curve detection was performed using the ramp mode with a Bioscope Catalyst (Bruker) equipped with a Nanoscope V controller and Nanoscope software version 8.15 (Supplementary Fig. 1a,b). The probes were standard pyramidal silicon nitride probes (MLCT model; Bruker) with triangular cantilevers at thicknesses of $0.59-0.61 \mu \mathrm{m}$, the probe's spring constant of $0.3-0.5 \mathrm{~N} \mathrm{~m}^{-1}$ (for QNM mode) or $0.1 \mathrm{~N} \mathrm{~m}^{-1}$ (for ramp mode) and a tip radius of $20 \mathrm{~nm}$. For all measurements, an indentation depth of $20-75 \mathrm{~nm}$ and an indentation force of 3-16 $\mathrm{nN}$ were used to measure local wall elasticity. The probe's spring constant was calibrated before each measurement. The probe's spring constant was calibrated by the relative method on the Bruker's polystyrene test sample (PDMS-SOFT-2 with 3.5 megapascals (Mpa)) for QNM measurement (Supplementary Fig. 1f), and was calibrated by thermal tuning for ramp mode measurement. The QNM mode can scan the entire surface to obtain elastic modulus values at high resolution. For our samples, we obtained over 65,000 elastic modulus values for a 256 pixel $\times 256$-pixel (equal to $20 \times 20 \mu \mathrm{m}^{2}$ or $10 \times 10 \mu \mathrm{m}^{2}$ ) area for every scan image. The three-dimensional elasticity maps in Fig. 1 and Supplementary Figs. 5 and 6 were obtained by the QNM mode. The QNM mode could detect a difference over the cell walls compared to the middle of the cells, as shown before ${ }^{5}$. In comparison, the ramp mode can be used to obtain the absolute elastic modulus of selected discrete locations ${ }^{31}$.

All measurements were carried out under water at room temperature to prohibit cell plasmolysis. Tomato leaf $\mathrm{P}_{2}$ and $\mathrm{P}_{3}$ primordia were detached from the shoot apex with syringe tips, tilted to the appropriate position and adhered to a Petri dish using nail polish. Shoot apexes, including $\mathrm{P}_{1}$ primordia, were adhered to a Petri dish with $\mathrm{P}_{1}$ primordia adjusted vertical to the probe (Fig. 1a). For the ramp mode, we chose the contact mode and only detected the surfaces that were flat and normal to the probes. For each sample type, we examined at least 24 cells in six leaf samples. Force curves were obtained 20 times at each point. Data were analysed with Nanoscope Analysis version 1.8. Raw AFM measurements are provided in Supplementary Table 2 .

Immunohistochemistry. For immunolocalization of de-methyl-esterification of HG, tomato or Arabidopsis shoot apices were fixed in methanol under vacuum and embedded in Steedman's wax composed of PEG 400 distearate and 1-hexadecanol (Sigma-Aldrich). After rehydration, $6 \mu \mathrm{m}$ sections were pretreated for $1 \mathrm{hr}$ with $2 \%$ BSA in PBS (for LM19, LM20 and JIM5 antibodies) or T/Ca/S (20 mM Tris-HCl, $1 \mathrm{mM} \mathrm{CaCl}_{2}, 150 \mathrm{mM} \mathrm{NaCl}, \mathrm{pH} 8.2$, for the $2 \mathrm{~F} 4$ antibody) buffer and incubated overnight with the antibody hybridoma supernatant (PlantProbes, Leeds, United Kingdom) diluted 1:500 in buffer containing 0.1\% (w/v) BSA. After three washes in buffer with $0.1 \%(\mathrm{v} / \mathrm{v})$ Tween 20 , sections were incubated for $1 \mathrm{hr}$ with secondary antibody Alexa Fluor 488 donkey anti-mouse IgG or Alexa Fluor 546 goat anti-rat IgG (Life Technologies, Carlsbad, CA, United States), diluted 1:1,000 in buffer supplemented with $0.1 \%(\mathrm{w} / \mathrm{v})$ BSA. After additional rinses in buffer plus $0.1 \%(\mathrm{v} / \mathrm{v})$ Tween 20, sections were mounted in ProLong Antifade (Life Technologies) under cover slips and examined using a Nikon $\mathrm{Al}^{+}$confocal laser scanning microscope. For the positive control for immunolocalization of de-methylesterification of $\mathrm{HG}$, sections on the slides were treated in buffer containing $0.05 \mathrm{M}$ $\mathrm{NaOH}$ for $30 \mathrm{~min}$ at $4{ }^{\circ} \mathrm{C}$ before the $2 \%$ BSA blocking step. We also included no primary antibody controls.

Micromanipulation. Local leaf treatment was performed as described before ${ }^{15,28}$ For PME treatment, Sephacryl HR S300 beads (GE Healthcare Life Sciences, Chicago, IL, United States) with an average particle size of $47 \mu \mathrm{m}$ were loaded with citrus PME (Sigma-Aldrich) by incubation ${ }^{15}$. Denatured PME was obtained by heating at $100^{\circ} \mathrm{C}$ for $20 \mathrm{~min}$ and overnight heating at $70^{\circ} \mathrm{C}$. One to three beads were picked up with forceps and positioned on each leaf primordium. This step drained the beads through capillarity on the forceps. For EGCG treatment, $50 \mathrm{mM}$ stock solutions of EGCG (Sigma-Aldrich) in DMSO were dissolved in lanolin (Sigma-Aldrich) prewarmed at $50^{\circ} \mathrm{C}$ to a final concentration of $10 \mathrm{mM}$. The paste was manually administered to cultured tomato leaf primordia with syringe tips ${ }^{28}$.

Construction of transgenic plants. The $p A S 2:: P M E 5$ construct contains the endogenous AS2 promoter (3,303 bp upstream of the start codon and $18 \mathrm{bp}$ of the N-terminal AS2 coding region) and the endogenous PME5 coding sequence (1,089 bp downstream of the start codon). pFIL::PMEI3 contains the endogenous FIL promoter (6,011 bp upstream of the start codon) and the endogenous PMEI3 coding sequence (618 bp downstream of the start codon). pATML1::PME5 and pATML1::PMEI3 contain the endogenous ATML1 promoter $(3,428$ bp upstream of the site 1,597 bp before the start codon $)^{59}$ and the PME5 or PMEI3 coding sequence. These constructs were introduced into the Col- 0 wild-type, rev- 6 and as2-2 plants using Agrobacterium-mediated transformation. Transformants were selected on MS plates containing basta, and at least 24 independent stable transformants were characterized for each construct.

RT-qPCR. PME- or EGCG-treated $\mathrm{P}_{4 / 5}$ primordia were separately collected according to morphological polarity phenotype $5 \mathrm{~d}$ after treatment, as described above. Mock-treated primordia were collected in parallel. Total RNA was extracted from each group of five primordia of the same treatment and phenotype using the RNeasy Micro kit (Qiagen, Hilden, Germany). First-strand complementary DNA (cDNA) synthesis was performed with about $0.5 \mu \mathrm{g}$ total RNA using TransScript One-Step gDNA Removal and cDNA Synthesis SuperMix (TransGen, Beijing, China) and 22-mer oligo(dT) primers according to the manufacturer's instructions. RT-qPCR was performed on a Bio-Rad CFX96 real-time PCR detection system with the KAPA SYBR FAST qPCR kit (KAPA Biosystems, Wilmington, MA, United States) and gene-specific primers (Supplementary Table 3). Relative expressions of PME/EGCG-treated samples were measured by RT-qPCR compared with mock-treated samples of the same batch, and all their expressions were normalized against the expression of LeActin (Solyc03g078400), which is a widely used internal reference control gene with stable expression, of the same sample.

In situ hybridization. Details of methods used for fixation of plants, embedding in paraffin and in situ hybridization can be found at http://www.its.caltech. $\mathrm{edu} / \sim$ plantlab/protocols/insitu.html. Sections ( $8 \mu \mathrm{m}$ thick) were cut with a Leica RM2255 rotary microtome. The FIL probe contains 612 bp of FIL cDNA amplified by PCR with primers 5'-ATGTCTATGTCGTCTATGTC-3' and 5'-CTGTTGGGGCATGTTGGTTT-3'; the AS2 probe contained $557 \mathrm{bp}$ of AS2 cDNA amplified by PCR with primers 5'-CTCCTCCAACATCAGCTTCG-3' and 5'-ATCAATTAAGAGAGCAAGTCCATAA-3'; the ATML1 probe contained $687 \mathrm{bp}$ of $A T M L 1 \mathrm{cDNA}$ amplified by PCR with primers 5'-GGATGTGAAT CAATGGTCTAGTGTG-3' and 5'-GTTCCCACTATTGACTCGAAGCA-3'; the LeFIL probe contained $657 \mathrm{bp}$ of LeFIL cDNA amplified by PCR with primers 5'-ATGTCGTCTTCATCTGCTGCTTTTGC-3' and 5'-TCAGTAAGGAGATACA CCAATGTTTGCTGG-3'; and the LeREV probe contained 1,297 bp of LeREV cDNA amplified by PCR with primers 5'-AAGTTGTGAATCAGTGGTAACC-3' and 5'-ATCAATAGGGGCAAAAACTAG-3'. All PCR amplicons were cloned into the pEASY-Blunt cloning vector (TransGen) for in vitro transcription.

Mechanical modelling. Morphological evolution of the leaf was predicted using a $2 \mathrm{D}$ mechanical model. The model assumes for simplicity that a leaf at its early $\left(\mathrm{P}_{1}\right)$ morphogenesis stage is a cylindrical disk, with the epidermis as its perimeter and the inner cells as its interior (Supplementary Fig. 7a). As detailed measurements for the interaction between the inner cells and the epidermis-that is, the squeezing forces-are currently lacking, we introduced in our model a small cushion zone that separates the inner cells from the epidermis. Dynamic growth of the inner cells is governed by the Hamiltonian potential energy equation, which should evolve within the region around the epidermis. Besides, a 'short-axis' rule was also introduced for treating the division of inner cells ${ }^{60}$. The viscoelastic property of the epidermis was modelled by the generalized Maxwell element (Supplementary Fig. 7b), whose creeping behaviour obeys the dynamic equilibrium equation under the first boundary condition. Multiple regulating parameters were included in numerical simulations (Supplementary Table 4), which present the mechanical parameters of cell walls in the dimensionless form. $E_{\text {stiff }} / E_{\text {soft }}$ and $\lambda_{\text {stiff }} / \lambda_{\text {soft }}$ represented the mechanical difference of stiff and soft inner cells and epidermis, respectively. Sensitivity analysis of key parameters in the model was performed in Supplementary Fig. 7c,d. To numerically solve our hybrid model, an in-house algorithm that combines the stochastic energy minimization method with the determinative finite element method was developed. For details, please refer to the Supplementary Methods. Stress and strain dynamics of the epidermal layer upon different relaxation cycles at different stages of leaf primordia were shown in Supplementary Fig. 7e-h.

Data availability. The data that support the findings of this study are available from the corresponding authors upon request. 
Received: 26 August 2016; Accepted: 28 July 2017;

Published online: 04 September 2017

\section{References}

1. Sablowski, R. Coordination of plant cell growth and division: collective control or mutual agreement? Curr. Opin. Plant Biol. 34, 54-60 (2016).

2. Lecuit, T. \& Lenne, P. F. Cell surface mechanics and the control of cell shape, tissue patterns and morphogenesis. Nat. Rev. Mol. Cell Biol. 8, 633-644 (2007).

3. Sampathkumar, A., Yan, A., Krupinski, P. \& Meyerowitz, E. M. Physical forces regulate plant development and morphogenesis. Curr. Biol. 24, R475-R483 (2014)

4. Louveaux, M., Julien, J. D., Mirabet, V., Boudaoud, A. \& Hamant, O. Cell division plane orientation based on tensile stress in Arabidopsis thaliana. Proc. Natl Acad. Sci. USA 113, E4294-E4303 (2016).

5. Sampathkumar, A. et al. Subcellular and supracellular mechanical stress prescribes cytoskeleton behavior in Arabidopsis cotyledon pavement cells. eLife 3, e01967 (2014).

6. Peaucelle, A. et al. Pectin-induced changes in cell wall mechanics underlie organ initiation in Arabidopsis. Curr. Biol. 21, 1720-1726 (2011).

7. Hervieux, N. et al. A mechanical feedback restricts sepal growth and shape in Arabidopsis. Curr. Biol. 26, 1019-1028 (2016).

8. Braam, J. In touch: plant responses to mechanical stimuli. New Phytol. 165, 373-389 (2005).

9. Gibson, W. T. et al. Control of the mitotic cleavage plane by local epithelial topology. Cell 144, 427-438 (2011)

10. Boudon, F. et al. A computational framework for 3D mechanical modeling of plant morphogenesis with cellular resolution. PLoS Comput. Biol. 11, e1003950 (2015)

11. Cosgrove, D. J. Wall extensibility: its nature, measurement and relationship to plant cell growth. New Phytol. 124, 1-23 (1993).

12. Cosgrove, D. J. Growth of the plant cell wall. Nat. Rev. Mol. Cell Biol. 6, 850-861 (2005).

13. Wolf, S., Hematy, K. \& Hofte, H. Growth control and cell wall signaling in plants. Annu. Rev. Plant Biol. 63, 381-407 (2012).

14. Peaucelle, A., Braybrook, S. \& Hofte, H. Cell wall mechanics and growth control in plants: the role of pectins revisited. Front. Plant. Sci. 3, 121 (2012).

15. Peaucelle, A. et al. Arabidopsis phyllotaxis is controlled by the methylesterification status of cell-wall pectins. Curr. Biol. 18, 1943-1948 (2008).

16. Ali, O., Mirabet, V., Godin, C. \& Traas, J. Physical models of plant development. Annu. Rev. Cell Dev. Biol. 30, 59-78 (2014).

17. Waites, R. \& Hudson, A. phantastica: a gene required for dorsoventrality of leaves in Antirrhinum majus. Development 121, 2143-2154 (1995).

18. Barton, M. K. Twenty years on: the inner workings of the shoot apical meristem, a developmental dynamo. Dev. Biol. 341, 95-113 (2010).

19. Bowman, J. L. \& Floyd, S. K. Patterning and polarity in seed plant shoots. Annu. Rev. Plant Biol. 59, 67-88 (2008).

20. Braybrook, S. A. \& Kuhlemeier, C. How a plant builds leaves. Plant Cell 22, 1006-1018 (2010)

21. Efroni, I., Eshed, Y. \& Lifschitz, E. Morphogenesis of simple and compound leaves: a critical review. Plant Cell 22, 1019-1032 (2010).

22. Husbands, A. Y., Chitwood, D. H., Plavskin, Y. \& Timmermans, M. C. Signals and prepatterns: new insights into organ polarity in plants. Genes Dev. 23, 1986-1997 (2009)

23. Xu, L., Yang, L. \& Huang, H. Transcriptional, post-transcriptional and post-translational regulations of gene expression during leaf polarity formation. Cell Res. 17, 512-519 (2007).

24. Emery, J. F. et al. Radial patterning of Arabidopsis shoots by class III HD-ZIP and KANADI genes. Curr. Biol. 13, 1768-1774 (2003).

25. Iwakawa, H. et al. The ASYMMETRIC LEAVES2 gene of Arabidopsis thaliana, required for formation of a symmetric flat leaf lamina, encodes a member of a novel family of proteins characterized by cysteine repeats and a leucine zipper. Plant Cell Physiol. 43, 467-478 (2002).

26. Siegfried, K. R. et al. Members of the YABBY gene family specify abaxial cell fate in Arabidopsis. Development 126, 4117-4128 (1999).

27. Sussex, I. M. Experiments on the cause of dorsiventrality in leaves. Nature 167, 651-652 (1951).

28. Qi, J. et al. Auxin depletion from leaf primordia contributes to organ patterning. Proc. Natl Acad. Sci. USA 111, 18769-18774 (2014).

29. Braybrook, S. A. \& Peaucelle, A. Mechano-chemical aspects of organ formation in Arabidopsis thaliana: the relationship between auxin and pectin. PLoS ONE 8, e57813 (2013).

30. Cleland, R. Cell wall extension. Annu. Rev. Plant Physiol. 22, 197-222 (1971).

31. Milani, P. et al. In vivo analysis of local wall stiffness at the shoot apical meristem in Arabidopsis using atomic force microscopy. Plant J. 67, 1116-1123 (2011).
32. Verhertbruggen, Y., Marcus, S. E., Haeger, A., Ordaz-Ortiz, J. J. \& Knox, J. P. An extended set of monoclonal antibodies to pectic homogalacturonan. Carbohydr. Res. 344, 1858-1862 (2009).

33. Clausen, M. H., Willats, W. G. \& Knox, J. P. Synthetic methyl hexagalacturonate hapten inhibitors of anti-homogalacturonan monoclonal antibodies LM7, JIM5 and JIM7. Carbohydr. Res. 338, 1797-1800 (2003).

34. Liners, F., Thibault, J. F. \& Van Cutsem, P. Influence of the degree of polymerization of oligogalacturonates and of esterification pattern of pectin on their recognition by monoclonal antibodies. Plant Physiol. 99, 1099-1104 (1992).

35. Peaucelle, A., Wightman, R. \& Hofte, H. The control of growth symmetry breaking in the Arabidopsis hypocotyl. Curr. Biol. 25, 1746-1752 (2015).

36. Hayashi, K. et al. Rational design of an auxin antagonist of the SCF(TIR1) auxin receptor complex. ACS Chem. Biol. 7, 590-598 (2012).

37. Krogan, N. T. \& Berleth, T. A dominant mutation reveals asymmetry in MP/ARF5 function along the adaxial-abaxial axis of shoot lateral organs. Plant Signal. Behav. 7, 940-943 (2012).

38. McConnell, J. R. et al. Role of PHABULOSA and PHAVOLUTA in determining radial patterning in shoots. Nature 411, 709-713 (2001).

39. Abley, K. et al. An intracellular partitioning-based framework for tissue cell polarity in plants and animals. Development 140, 2061-2074 (2013).

40. Kuchen, E. E. et al. Generation of leaf shape through early patterns of growth and tissue polarity. Science 335, 1092-1096 (2012).

41. Lawrence, P. A., Struhl, G. \& Casal, J. Planar cell polarity: one or two pathways? Nat. Rev. Genet. 8, 555-563 (2007).

42. Stopper, G. F. \& Wagner, G. P. Of chicken wings and frog legs: a smorgasbord of evolutionary variation in mechanisms of tetrapod limb development. Dev. Biol. 288, 21-39 (2005).

43. Kennaway, R., Coen, E., Green, A. \& Bangham, A. Generation of diverse biological forms through combinatorial interactions between tissue polarity and growth. PLoS Comput. Biol. 7, e1002071 (2011).

44. Merks, R. M., Guravage, M., Inze, D. \& Beemster, G. T. VirtualLeaf: an open-source framework for cell-based modeling of plant tissue growth and development. Plant Physiol. 155, 656-666 (2011).

45. Kierzkowski, D. et al. Elastic domains regulate growth and organogenesis in the plant shoot apical meristem. Science 335, 1096-1099 (2012).

46. Juge, N. Plant protein inhibitors of cell wall degrading enzymes. Trends Plant Sci. 11, 359-367 (2006)

47. Tian, C. et al. An organ boundary-enriched gene regulatory network uncovers regulatory hierarchies underlying axillary meristem initiation. Mol. Syst. Biol. 10, 755 (2014)

48. Talbert, P. B., Adler, H. T., Parks, D. W. \& Comai, L. The REVOLUTA gene is necessary for apical meristem development and for limiting cell divisions in the leaves and stems of Arabidopsis thaliana. Development 121, 2723-2735 (1995).

49. Nakata, M. et al. Roles of the middle domain-specific WUSCHEL-RELATED HOMEOBOX genes in early development of leaves in Arabidopsis. Plant Cell 24, 519-535 (2012).

50. Sessions, A., Weigel, D. \& Yanofsky, M. F. The Arabidopsis thaliana MERISTEM LAYER 1 promoter specifies epidermal expression in meristems and young primordia. Plant J. 20, 259-263 (1999).

51. Coen, E., Rolland-Lagan, A. G., Matthews, M., Bangham, J. A. \& Prusinkiewicz, P. The genetics of geometry. Proc. Natl Acad. Sci. USA 101, 4728-4735 (2004).

52. Wang, J., Lu, D., Mao, D. \& Long, M. Mechanomics: an emerging field between biology and biomechanics. Protein Cell 5, 518-531 (2014).

53. Kutschera, U. \& Niklas, K. J. The epidermal-growth-control theory of stem elongation: an old and a new perspective. J. Plant Physiol. 164, 1395-1409 (2007).

54. Reinhardt, D., Frenz, M., Mandel, T. \& Kuhlemeier, C. Microsurgical and laser ablation analysis of leaf positioning and dorsoventral patterning in tomato. Development 132, 15-26 (2005).

55. McConnell, J. R. \& Barton, M. K. Leaf polarity and meristem formation in Arabidopsis. Development 125, 2935-2942 (1998).

56. Semiarti, E. et al. The ASYMMETRIC LEAVES2 gene of Arabidopsis thaliana regulates formation of a symmetric lamina, establishment of venation and repression of meristem-related homeobox genes in leaves. Development 128, 1771-1783 (2001).

57. Heisler, M. G. et al. Patterns of auxin transport and gene expression during primordium development revealed by live imaging of the Arabidopsis inflorescence meristem. Curr. Biol. 15, 1899-1911 (2005).

58. Wang, Y. et al. The stem cell niche in leaf axils is established by auxin and cytokinin in Arabidopsis. Plant Cell 26, 2055-2067 (2014).

59. Takada, S. \& Jurgens, G. Transcriptional regulation of epidermal cell fate in the Arabidopsis embryo. Development 134, 1141-1150 (2007).

60. Shapiro, B. E., Tobin, C., Mjolsness, E. \& Meyerowitz, E. M. Analysis of cell division patterns in the Arabidopsis shoot apical meristem. Proc. Natl Acad. Sci. USA 112, 4815-4820 (2015). 


\section{Acknowledgements}

We thank K.-I. Hayashi (Okayama University of Science) for providing auxinole, N. Li (Institute of Mechanics, Chinese Academy of Sciences) and Z. Huang (Bruker Nano Surfaces Business, Beijing) for assistance with AFM measurement, the Core Facilities of Life Sciences of Peking University for use of the TEM and S.-N. Bai (Peking University) and S. Poethig (University of Pennsylvania) for discussions. This work was supported by National Natural Science Foundation of China grants 31430010 and 31627804, National Basic Research Program of China (973 Program) grants 2014CB943500 and 2011CB710900, National Key Research and Development Program of China grant 2016YFA0501601, the National Program for Support of Top-Notch Young Professionals, China Postdoctoral Science Foundation grant 2015M570171 and the State Key Laboratory of Plant Genomics.

\section{Author contributions}

Y.J. conceived and designed experiments. J.Q. and B.W. carried out most of the experiments. S.F., S.L. and M.L. carried out numerical simulations.
C.G. contributed to phenotypic analysis. X.Z. and D.Q. contributed to AFM experiments. Y.H. performed TEM experiments. Y.Z. and C.L. provided materials/reagents. Y.J. and M.L. wrote the manuscript, with contributions from all the authors.

\section{Competing interests}

The authors declare no competing financial interests.

\section{Additional information}

Supplementary information is available for this paper at doi:10.1038/s41477-0170008-6.

Reprints and permissions information is available at www.nature.com/reprints. Correspondence and requests for materials should be addressed to M.L. or Y.J. Publisher's note: Springer Nature remains neutral with regard to jurisdictional claims in published maps and institutional affiliations. 